\title{
DPP4 Inhibitors and COVID-19-Holy Grail or Another Dead End?
}

\author{
Alicja Krejner-Bienias ${ }^{1} \cdot$ Katarzyna Grzela $^{2} \cdot$ Tomasz Grzela $^{1}$ (1) \\ Received: 1 August 2020 / Accepted: 10 December 2020 / Published online: 2 February 2021 \\ (c) L. Hirszfeld Institute of Immunology and Experimental Therapy, Wroclaw, Poland 2021
}

\begin{abstract}
A novel coronavirus disease, COVID-19, has emerged as a global public health issue. Clinical course of disease significantly correlates with the occurrence of some comorbidities, among them type 2 diabetes. According to recent structural studies the dipeptidyl peptidase 4, a key molecule in the pathophysiology of diabetes, may influence the course of COVID-19. Since DPP4 inhibitors, gliptins, are widely used in diabetes patients, the exact role of DPP4 modulation in SARS-CoV-2 infection, at least in that group, urgently needs to be clarified. In this short review, we discuss this issue with more detail.
\end{abstract}

Keywords SARS-CoV-2 $\cdot$ COVID-19 $\cdot$ DPP4 $\cdot$ Gliptins $\cdot$ Diabetes

\section{Introduction}

A novel coronavirus disease 2019 (COVID-19), with $60,011,873$ identified infected patients, and 1,412,502 lethal cases registered until November 24, 2020, has appeared a most urgent global public health issue (World Health Organization 2020). The disease is caused by the severe acute respiratory syndrome coronavirus-2 (SARS-CoV-2), a relatively large RNA virus, with virions of $140-200 \mathrm{~nm}$ in diameter, belonging to the broad family of coronaviruses. According to current classification, with genetic similarity nearly $80 \%$, SARS-CoV-2 is considered as a strain of SARS-CoV, which was implicated with SARS outbreak in 2002-2004 (Gorbalenya et al. 2020; Lu et al. 2020). At least seven representatives of the coronaviruses family, including both, SARS-CoV and -CoV-2, the Middle East respiratory syndrome (MERS)-CoV, and the next four-NL63, 229E, HKU1 and OC43, are responsible for broad spectrum of illnesses in human-from common cold to severe multi-organ failure.

The clinical course of COVID-19 depends on age, general patients' condition, and comorbidities. While the average

Tomasz Grzela

tomasz.grzela@wum.edu.pl; tomekgrzela@gmail.com

1 Department of Histology and Embryology, Medical University of Warsaw, Chalubinskiego 5, 02-004 Warsaw, Poland

2 Department of Pediatric Pulmonology and Allergy, Medical University of Warsaw, Warsaw, Poland fatality rate in COVID-19 concerns approx. $2.35 \%$ of diagnosed/confirmed cases for the whole population, it increases almost nine-fold (22.8\%) for individuals in age group above 70 (World Health Organization 2020).

Besides the most prevalent and most studied cardiovascular diseases (CVD) with hypertension, found in $29.5 \%$ infected patients, the next among comorbidities, the diabetes, mainly type 2 (T2D), affects approximately $9.7 \%$ of all individuals with COVID-19 (Yang et al. 2020). Although such frequency is similar to that in general population, nevertheless, when compared between severe and nonsevere COVID-19 course, T2D was even threefold higher in patients, who required admission to intensive care units, then those with the mild course of SARS-CoV-2 infection (Roncon et al. 2020; Yang et al. 2020).

The reason for poorer outcome in diabetic patients remains not fully explained. Certainly, it may be due to the impaired immune response to SARS-CoV-2, that may result from chronic hyperglycaemia and a low-grade chronic inflammation associated with T2D (Berbudi et al. 2020). On the other hand, patients with T2D constitute a highly heterogeneous population in terms of already mentioned comorbidities, such as CVD and hypertension, but also obesity, or diabetic complications. Obviously, each of them alone could increase the risk of severe course of COVID-19 (Hussain et al. 2020; Yang et al. 2020). However, recent studies may suggest some alternative explanation. 


\section{SARS-CoV-2 Invasion}

Coronavirus invasion requires the interaction between viral spike (S) protein and the specific receptor on the surface of the host cell membrane. SARS-CoV and SARS-CoV-2 utilize angiotensin-converting enzyme (ACE)-2 as their main entry receptor, whereas MERS-CoV infects human cells by binding to another membrane-bound protease-dipeptidyl peptidase (DPP)4 (Li et al. 2003, 2020a; Raj et al. 2013). The critical step, which is necessary for target cell infection, involves the priming of viral $\mathrm{S}$ protein. The priming constitutes the cleavage of spike protein by cellular serine proteases-mainly transmembrane protease serine- 2 and, in lesser extent, endosomal cysteine proteases-cathepsin B and $\mathrm{L}$, that enables fusion of viral and cellular membranes and virus entry into target cell (Hoffman et al. 2020).

Interestingly, in silico modelling of the homo-trimer structure of SARS-CoV-2 spike protein predicted that its $\mathrm{S} 1$ domain, in addition to ACE-2, may potentially interact also with the human DPP4 (Fig. 1). The analysis of molecular models of SARS-CoV-2 S protein and DPP4 has shown a large interface between both proteins, enabling a tight connection between the S1 domain loops and DPP4 surface (Vankadari and Wilce 2020). The calculated affinity of SARS-CoV-2 to ACE-2 was stronger, when compared to DPP4, suggesting the priority for the SARS-CoV-2-ACE-2 binding, and its leading function in viral invasion. Noteworthy, while no potential interactions of SARS-CoV-S with DPP4 and MERS-CoV with ACE-2 (with calculated freeenergy values $>0 \mathrm{kcal} / \mathrm{mol}$ ) were predicted, among the three analyzed models of coronaviruses, only SARS-CoV-2-S

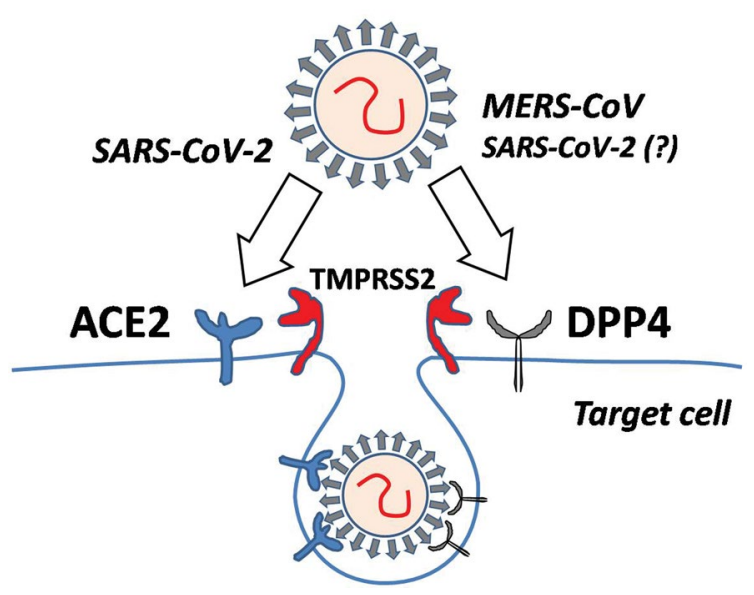

Fig. 1 Two pathways of coronavirus invasion, involving membranebound angiotensin-converting enzyme (ACE)-2 or dipeptidyl peptidase (DPP) 4 molecules. SARS-CoV-2-severe acute respiratory syndrome coronavirus-2, MERS-CoV-Middle East respiratory syndrome coronavirus. TMPRSS2-transmembrane protease serine-2. The detailed description in text could theoretically bind to both ACE-2 and DPP4 (Li et al. 2020b). This might, hypothetically, enable the easier spreading of SARS-CoV-2 infection in broader spectrum of tissues and could explain tropism either to respiratory and gastrointestinal epithelia.

Surprisingly, the marginal role, if any, of DPP4 in SARSCov-2 infection was concluded based on the results of experiments with hamster cells transiently transfected with human DPP4 (Hoffman et al. 2020). Similar conclusion originates from another artificial system with human DPP4 expressed in insect cells (Xi et al. 2020). However, both aforementioned experimental models have some limitations, which do not allow to exclude the role of DPP4 in SARS-CoV-2 invasion definitely.

Therefore, the presumable involvement of DPP4 as an accessory entry receptor for SARS-CoV-2 and its role in the course of COVID-19 still may be of noticeable clinical relevance (Bassendine et al. 2020).

\section{DPP4 in Health and Disease}

DPP4, also known as the cluster of differentiation 26 (CD26), is a $110 \mathrm{kDa}$ aminopeptidase, widely expressed as a homodimer on the surface of many cell types, including respiratory epithelia. It interacts with various cellular and extracellular proteins. Due to its protease properties, DPP4 cleaves a wide range of chemokines, immunomodulatory proteins and peptide hormones, preferentially digesting molecules with alanine or proline residue in the penultimate amino-terminal position (Lambeir et al. 2003).

It has been proven that DPP4 plays a key role in glucose metabolism by the degradation of incretins, such as glucagon-like peptide 1 (GLP-1) and glucose-dependent insulinotropic polypeptide (GIP). Since incretins are responsible for increase of insulin secretion and decrease of blood glucose level, their degradation by DPP4 is important mechanism of glucose homeostasis. Therefore, overexpression and increased activity of DPP4 are associated with diabetes, and obesity-induced metabolic syndrome (Bassendine et al. 2020; Lamers et al. 2011). DPP4 has been found to be involved in pathomechanisms of different processes, e.g. angiogenesis and carcinogenesis. Some studies suggest its role in the development of fibrosis of various organs, such as kidneys and liver (Kaji et al. 2014; Panchapakesan and Pollock 2015).

While the list of target molecules for DPP4 includes several chemokines and other immunoregulatory proteins, this peptidase is also involved in modulation of immune response, e.g. in course of viral infections. It has been found that patients with chronic hepatitis $\mathrm{C}$ virus infection have elevated serum levels of C-X-C motif chemokine ligand 10 (CXCL10), also known as interferon gamma-induced protein 
10. This chemokine, after its DPP4-mediated transformation to amino-terminally truncated form, may affect inflammatory reaction working as an antagonist for specific receptor CXCR3 (Bassendine et al. 2020; Casrouge et al. 2011).

Besides that enzymatic activity, DPP4 may also reveal some non-enzymatic interactions. Among them, the binding of adenosine deaminase (ADA) to DPP4 seems to be critical in providing co-stimulatory signals to $\mathrm{T}$ cells (Röhrborn et al. 2015). Increased DPP4-ADA results in increased degradation of adenosine, the potent suppressor of $\mathrm{T}$ lymphocytes, thus increasing proliferation of these cells. Hence, DPP4 may modify both acquired and innate immune responses (Bassendine et al. 2020; Klemann et al. 2016). Interestingly, the co-stimulatory function of DPP4 in the activation of human memory $\mathrm{T}$ lymphocytes does not necessarily require participation of ADA (De Meester et al. 1995).

When considering immunoregulatory properties of DPP4, including aforementioned DPP4-ADA-mediated immunomodulation, its increased levels may promote subclinical chronic inflammation and dysregulation of immune system. Due to such dysregulation, DPP4 may also indirectly support the development of COVID-19 in diabetic and obese patients. Possibly, it could explain more severe course of COVID-19 in these populations, with increased susceptibility to cytokine storm and fulminant multi-organ failure.

The knowledge regarding the involvement of DPP4related mechanisms in coronaviruses infections may be translated from animal studies focused on MERS-CoV, where DPP4 has been identified as the main entry receptor for this virus. Using transgenic mouse models, it has been proven that in animals expressing human DPP4, a high-fat diet induced hyperglycaemia and hyperinsulinemia, resembling human T2D, whereas mice lacking the gene for DPP4 were protected against obesity and insulin resistance. Noteworthy, diabetic mice infected with MERS-CoV developed more severe course of the disease and prolonged recovery than non-obese animals without human DPP4 (Conarello et al. 2003; Kulcsar et al. 2019). However, one has to note that these models have some limitations, at least in regard to DPP4-ADA-related immune dysregulation. The latter takes no place in mouse system, since mouse DPP4 does not bind ADA molecule (Abbott et al. 1999).

Besides T2D and obesity, the next clinically relevant risk factor, which appears to be associated with presumable link between DPP4 and susceptibility to SARS-CoV-2 infection, is the age of infected patients. Interestingly, in vitro studies revealed that DPP4 was selectively expressed on the surface of senescent, but not of proliferating diploid human fibroblasts (Kim et al. 2017). Furthermore, the activity of DPP4 molecule was higher in older individuals, compared to younger ones (Bassendine et al. 2020). The studies focused on interaction between DPP4 and MERS-CoV spike protein have revealed that this interaction seems to be age-dependent, as it may be modified by the level of DPP4 sialylation. The increased sialylation of DPP4 influences its surface charge, and thus may facilitate virus binding and entry into target cell. Noteworthy, it has been proven that in patients with age above 40 , the number of sialylated isoforms of DPP4 is significantly higher, as compared to younger individuals (Smith et al. 1998). Apart from the modulation of MERS-CoV-DPP4 interaction, aforementioned DPP4 hypersialylation-dependent phenomenon was suggested as an auxiliary mechanism, which seems to be involved in fusion of target cells with human immunodeficiency virus (HIV) (Smith et al. 1998). It is plausible that this interaction could play accessory role also in SARSCoV-2 infection. Nevertheless, this hypothesis still requires verification in further studies.

The last but not least is the increased susceptibility to coronavirus infection in patients with chronic diseases of respiratory system, which may be attributed to DPP4 up-regulation, too. The significant increase of both DPP4 mRNA and protein levels was observed in the respiratory tract epithelium of cigarette smokers and patients with chronic obstructive pulmonary disease, thus suggesting that these individuals are more susceptible especially to MERS-CoV infection (Seys et al. 2018), and possibly, also to SARS$\mathrm{CoV}-2$. However, the latter needs to be verified.

\section{Soluble DPP4}

The complexity of pathophysiological role of DPP4, particularly, its involvement in coronaviruses infections, may further be complicated by the fact that, besides the membranebound molecule, it may also exist as a soluble form (sDPP4). sDPP4 is cleaved from the cell membrane and released into circulation, still remaining enzymatically active. It has been found that sDPP4 was significantly higher in young, as compared to older individuals (Lamers et al. 2011). Noteworthy, serum levels of sDPP4 are altered in many pathophysiological conditions, including obesity and metabolic syndrome. They positively correlate with insulin resistance, body mass index and the size of adipocytes in subcutaneous and visceral fat (Lamers et al. 2011; Röhrborn et al. 2015).

According to Casrouge et al. (2018), major sources of sDPP4 are lymphocytes, and T cell activation accounts for the dynamic changes observed in its plasma concentration.

Since high level of surface DPP4/CD26 is characteristic for human population of $\mathrm{CD} 3{ }^{+} \mathrm{CD} 4{ }^{+} \mathrm{CD} 45 \mathrm{RO}^{+} \mathrm{T}$ helper/ memory lymphocytes, it is plausible that the soluble form of this molecule may enable memory cells to be more responsive to recall antigen. Interestingly, in vitro experiments have shown that sDPP4 may reveal some immunomodulatory properties and stimulates the proliferative response of human T lymphocytes, which is independent of both the 
enzyme activity and the ADA binding capability. Possibly, this could be due to interfering of SDPP4 with costimulatory function of its membrane form and thereby affecting signal transduction and providing protection from anergy or apoptosis of T cells (Yu et al. 2011).

In clinical studies, it has been found that the levels of SDPP4 are increased in patients with acute and chronic viral infections; hence, sDPP4 concentration might be used as a biomarker for inflammatory and infectious diseases (Andrieu et al. 2003; Casrouge et al. 2018).

Unexpectedly, in patients infected with MERS-CoV, the concentration of sDPP4 in plasma was significantly decreased and correlated with disease severity (Inn et al. 2018; Zhao et al. 2020). Recently, similar observation

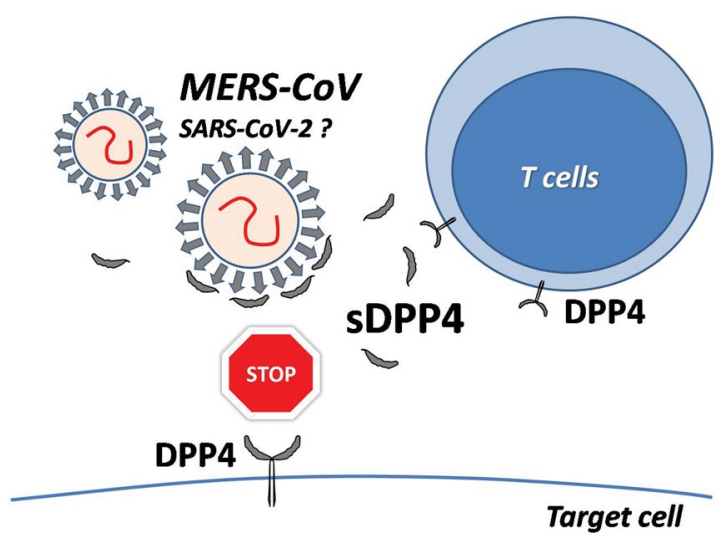

Fig. 2 Possible protective function of soluble DPP4 (sDPP4) in coronavirus infection. The circulating sDPP4 may coat virus spike proteins and thus prevent their interaction with membrane-bound DPP4 on the target cell. The detailed description in text was reported in patients with severe course of COVID-19 (Schlicht et al. 2020). However, it is unclear, whether sDPP4 reduction was a simple effect of lymphopenia, which is typical for patients infected with novel coronaviruses, or should rather be considered as an initial condition and presumable cause of increased susceptibility to MERS-CoV or SARS$\mathrm{CoV}-2$ infection. The latter option could be supported by data from in vivo experiments with transgenic mice overexpressing human DPP4, which also exhibited increased circulating levels of sDPP4. Intriguingly, these animals were relatively resistant to MERS-CoV infection and developed milder lung inflammation and reduced rates of mortality (Algaissi et al. 2019; Drucker 2020).

It is plausible that SDPP4 could act as a decoy molecule for $\mathrm{S}$ proteins of both coronaviruses. According to that, sDPP4 might block the binding of viral S protein to the cell surface, thus decreasing virulence of MERS-CoV and, possibly, SARS-CoV-2 (Fig. 2). The postulated sDPP4-mediated interference with $\mathrm{S}$ protein and membrane-bound DPP4 interaction could practically be used to modulate the infectious potential of MERS-CoV and, possibly, SARS-CoV-2.

Besides sDPP4, the protective function against coronavirus infections was also suggested for aforementioned adenosine deaminase. Indeed, ADA has been shown to be a competitive antagonist for DPP4-mediated entry of MERSCoV by blocking the binding of S protein to DPP4 molecule on the surface of target cell (Fig. 3) (Raj et al. 2014). However, the similar mode of protective ADA action in case of SARS-CoV-2 still needs to be confirmed.

Apart from ADA and aforementioned soluble DPP4, the function of membrane-bound form of this molecule may also be modified by novel group of synthetic DPP4 inhibitors
Fig. 3 The schematic structure of membrane-bound DPP4 dimer. The grey scale portions of DPP4 molecules represent their three main regionscystein-rich, glycosylation-rich, and catalytic. Numbers in ovals indicate the binding sites for: $1-$ adenosine deaminase (ADA), 2 spike proteins of coronaviruses, and 3-DPP4 inhibitors. The detailed description in text

\section{1 - Binding site for ADA \\ 2 - Binding site for MERS-CoV Spike protein / putative binding site for SARS-CoV-2Spike protein 3 - Binding site for gliptins}

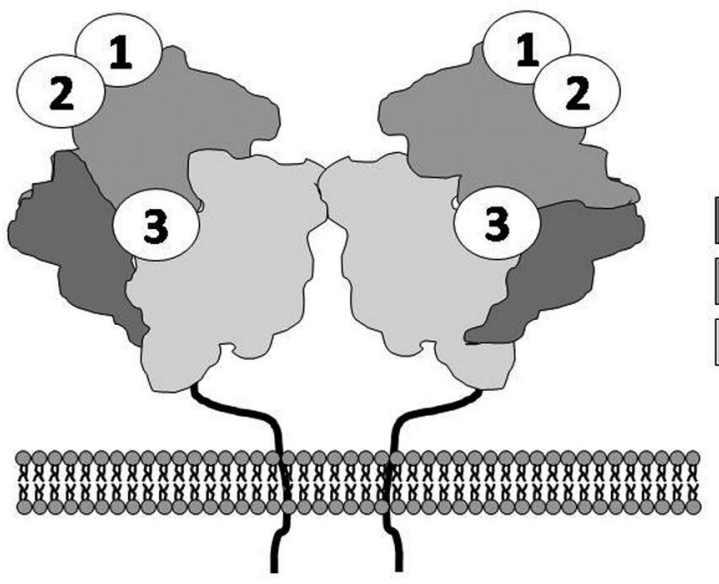

Cystein-rich region Glycosylation-rich region Catalytic region 
(DPP4i), also known as gliptins. Since gliptins are widely used as antidiabetic agents, their presumable involvement in the DPP4-related modulation of COVID-19 clinical course, at least in diabetic patients, was independently postulated by several authors in the beginning of April 2020 (Drucker 2020; Iacobellis 2020; Krejner-Bienias et al. 2020; Solerte et al. 2020a).

\section{DPP4 Inhibitors in COVID-19?}

Gliptins prevent the DPP4-mediated enzymatic degradation of incretins, including GLP-1 and GIP. Increased levels and prolonged half-life of incretins in T2D patients treated with DPP4i result in the inhibition of glucagon release, the stimulation of insulin secretion and the decrease of blood glucose. Noteworthy, DPP4i are body weight-neutral and present good safety and tolerability profiles (Gallwitz 2019). The gliptins are relatively new option in T2D treatment, as the first drug from this class, sitagliptin, was approved by the Food and Drug Administration in 2006 (Sesti et al. 2019).

Based on aforementioned considerations, it was suggested that DPP4i could be beneficial for patients with COVID-19, possibly by interfering with entry of the virus (Krejner-Bienias et al. 2020; Solerte et al. 2020a). However, in silico simulations concerning the molecular interaction between structural model of DPP4 and its inhibitors, or viral spike proteins of MERS-CoV and SARS-CoV-2, have clearly shown that binding sites for gliptins do not overlap with those for viral S proteins (Fig. 3) (Arulmozhiraja et al. 2016; Vankadari and Wilce 2020). Hence, this would exclude direct effect of gliptins on SARS-CoV-2 binding to DPP4 and cell infection.

Interestingly, based on the bioinformatics analysis of possible functional connections between sitagliptin and various cellular targets, Bardaweel et al. (2021) have proposed alternative influence of this drug on virus entry. The postulated indirect role of gliptins in modulation of SARS-CoV-2 endocytic pathway could result from their interference with interaction between DPP4 and caveolin-1, a scaffolding protein, necessary for endosomes formation (Bardaweel et al. 2021). However, although the protective role of one of DPP4i, teneligliptin, in DPP4-caveolin-dependent tissue injury has been confirmed in mouse model of diabetic kidney disease (Takagaki et al. 2019), so far there is no clear evidence regarding this mode of DPP4 $i$ action in patients with SARS-CoV-2 infection.

On the other hand, while the enzymatic action of DPP4 seems to be dispensable for viral infection, its inhibition may result in the up-regulation of this peptidase, similar to that found in ACE system (Adil et al. 2020). Based on this assumption, it is plausible that, using these drugs, patients with hypertension and/or diabetes may be more susceptible to severe COVID-19 course (Ding and Liang 2020).
Nevertheless, most recently, a large prospective cohort study with 8.3 million participants has proved that ACE inhibitors are safe and even reduce the risk of severe COVID-19 and its complications (Hippisley-Cox et al. 2020). Meanwhile, the clinical consequences of DPP4 inhibition by gliptins in COVID-19 patients still remain to be clarified.

The main issue to be taken into account is the general safety of gliptins use, i.e. whether potential benefits of these drugs would outweigh possible side effects, especially in COVID-19 patients. One has to consider the possibility that gliptins may affect immune response and thus increase the general risk of infection. This risk may be due to the wide expression of DPP4 on various cell types, including immune cells. In fact, the influence of gliptins on the immune response remains not fully understood. Extensive preclinical studies on mouse models using DPP4i did not demonstrate any evidence of impaired T cell-dependent immune responses (Vora et al. 2009). Moreover, the 28 days of administration of sitagliptin in healthy individuals had no effect on their lymphocyte and $\mathrm{T}$ cell subsets and did not affect plasma chemokine/cytokine levels or cytokines release by stimulation of peripheral blood mononuclear cells with either lipopolysaccharide or anti-CD3 antibody (Price et al. 2013). Also the long-term treatment with sitagliptin, assessed in the open label observational study of 34 subjects with T2D, did not affect levels of $\mathrm{CD}^{+}, \mathrm{CD}^{+}$, or NK cells. Either, the plasma levels of main cytokines did not differ after one-year therapy (Sromova et al. 2016).

Also the meta-analysis conducted by Yang et al. (2016) showed that the risk of infection in different systems, including respiratory tract infections, for DPP4i treatment was comparable to placebo. Similar results, confirming the safety of gliptins, were presented also by other researchers (Gooßen and Gräber 2012; Wvan der Zanden et al. 2015).

Noteworthy, 16-week treatment with sitagliptin did not affect selected inflammatory biomarkers even in HIV-positive individuals. Ninety patients on stable anti-retroviral therapy, without diabetes, were randomized to sitagliptinor placebo-receiving groups. Apart from decreased levels of CXCL10 in sitagliptin-treated patients, no differences between both groups were observed in total $\mathrm{CD} 4^{+}$and $\mathrm{CD} 8^{+}$ cells counts, as well as levels of C-reactive protein (CRP), interleukin (IL)-6, soluble CD14 (a marker of monocyte activation), soluble receptors for tumor necrosis factor-sTNFRI, and sTNF-RII, and sDPP4 (Dubé et al. 2019).

On the other hand, in another small study, it has been found that 12-week sitagliptin treatment of 22 patients with T2D caused a significant fall in their plasma concentrations of CRP and IL-6 (Makdissi et al. 2012). This observation might have practical importance, since recent data suggest that the inflammatory response plays a critical role in COVID-19. The serum levels of IL-6 and CRP seem to positively correlate with the severity of COVID-19 course, 
while inflammatory cytokine storm can lead to severe complications and death (Liu et al. 2020). Possibly, anti-inflammatory effect of sitagliptin could help to prevent and control cytokine storm in COVID-19 patients, being used alone, or more likely, in combination with other already used agents, like anti-IL-6 receptor antibody, tocilizumab, or dexamethasone (Atal and Fatima 2020; Kupferschmidt 2020).

It is obvious that the best way to evaluate, whether DPP4i may really be safe and useful in patients with SARS-CoV-2 infections, was the direct comparison of disease morbidity and severity among diabetic patients already treated with DPP4i, versus not receiving a such treatment.

In retrospective epidemiological study, Fadini et al. (2020) have analyzed group of 85 patients with T2D, hospitalized for COVID-19. Among them, only nine individuals used anti-DPP4 therapy prior to infection. Authors did not find any evidence that treatment with gliptins might affect hospitalization due to COVID-19 as compared to other T2D patients, without DPP4i. However, due to very low number of individuals included for analysis, the value of aforementioned study is strongly limited.

Interestingly, similar conclusion came from a large-scale French CORONADO (Coronavirus SARS-CoV-2 and Diabetes Outcomes) study. The investigators have assessed 1317 individuals with T2D and COVID-19, among them $284(21.6 \%)$ patients were treated with gliptins. Similarly to report by Fadini et al. (2020), the analysis did not indicate any significant impact of DPP4i therapy on COVID-19 course in diabetic patients (Scheen et al. 2020).

In contrast to aforementioned, the opposite conclusion was provided from the Italian multicenter trial-SIDIACO (Sitagliptin in Type 2 Diabetes and COVID-19). The study concerned 338 hospitalized T2D patients with COVID-19. At the time of hospitalization, patients were allocated into two treatment groups: sitagliptin and standard-of-care, or standard-of-care only, at a ratio 1:1. The clinical analysis has shown that the addition of sitagliptin to standard-ofcare treatment, when compared to standard-of-care only, was associated with significant improvement of clinical outcomes $(60 \%$ vs. $38 \%)$ and reduced mortality ( $18 \%$ vs $37 \%$ of deceased patients, respectively) (Solerte et al. 2020b). However, to clarify the exact mechanism of presumable beneficial effects of gliptins in COVID-19 patients, more data from ongoing clinical trials are required.

\section{Conclusion}

Despite extensive studies on COVID-19, there is no fully effective treatment available so far. Although structural models predict the tight binding between SARS-CoV-2 spike protein and human DPP4 on the cell surface, still there is no direct evidence to confirm the clinical relevance of such interaction. Certainly, the question, whether DPP4 inhibitors, gliptins, will emerge as a "holy grail" or just another dead end in COVID-19 research, may be important not only for T2D patients. However, since gliptins are widely used mainly to manage those patients, the exact role of DPP4 modulation in SARS-CoV-2 infection, at least in that group, urgently needs to be clarified.

Acknowledgements None.

Funding None.

\section{Compliance with ethical standards}

Conflict of interest None.

\section{References}

Abbott CA, McCaughan GW, Levy MT et al (1999) Binding to human dipeptidyl peptidase IV by adenosine deaminase and antibodies that inhibit ligand binding involves overlapping, discontinuous sites on a predicted beta propeller domain. Eur J Biochem 266:798-810. https://doi.org/10.1046/j.1432-1327.1999.00902.x

Adil MS, Narayanan SP, Somanath PR (2020) Isamiloride a promising cardiovascular medication to persist in the COVID-19 crisis? Drug DiscovTher 14:256-258. https://doi.org/10.5582/ ddt.2020.03070

Algaissi A, Agrawal AS, Han S et al (2019) Elevated human dipeptidyl peptidase 4 expression reduces the susceptibility of hDPP4 transgenic mice to Middle East Respiratory Syndrome coronavirus infection and disease. J Infect Dis 219:829-835. https://doi. org/10.1093/infdis/jiy574

Andrieu T, Thibault V, Malet I et al (2003) Similar increased serum dipeptidyl peptidase IV activity in chronic hepatitis $\mathrm{C}$ and other viral infections. J ClinVirol 27:59-68. https://doi.org/10.1016/ s1386-6532(02)00128-2

Arulmozhiraja S, Matsuo N, Ishitsubo E et al (2016) Comparative binding analysis of dipeptidyl peptidase IV (DPP-4) with antidiabetic drugs - An ab initio fragment molecular orbital study. PLoS ONE 11:e0166275. https://doi.org/10.1371/journal.pone.0166275

Atal S, Fatima Z (2020) IL-6 inhibitors in the treatment of serious COVID-19: A promising therapy? Pharmaceut Med 34:223-231. https://doi.org/10.1007/s40290-020-00342-z

Bardaweel SK, Hajjo R, Sabbah DA (2021) Sitagliptin: a potential drug for the treatment of COVID-19? Acta Pharm 71:175-184. https:// doi.org/10.2478/acph-2021-0013

Bassendine MF, Bridge SH, McCaughan GW et al (2020) COVID19 and comorbidities: A role for dipeptidylpeptidase 4 (DPP4) in disease severity? J Diabetes 12:649-658. https://doi. org/10.1111/1753-0407.13052

Berbudi A, Rahmadika N, Tjahjadi AI et al (2020) Type 2 diabetes and its impact on the immune system. Curr Diabetes Rev 16:442-449. https://doi.org/10.2174/1573399815666191024085838

Casrouge A, Decalf J, Ahloulay M et al (2011) Evidence for an antagonist form of the chemokine CXCL10 in patients chronically infected with HCV. J Clin Invest 121:308-317. https://doi. org/10.1172/JCI40594

Casrouge A, Sauer AV, Barreira da Silva R et al (2018) Lymphocytes are a major source of circulating soluble dipeptidyl peptidase 4 . ClinExpImmunol 194:166-179. https://doi.org/10.1111/cei.13163 
Conarello SL, Li Z, Ronan J et al (2003) Mice lacking dipeptidyl peptidase IV are protected against obesity and insulin resistance. Proc Natl AcadSci USA 100:6825-6830. https://doi.org/10.1073/ pnas.0631828100

De Meester IA, Kestens LL, Vanham GL et al (1995) Costimulation of CD4+ and CD8+ T cells through CD26: the ADA-binding epitope is not essential for complete signaling. J LeukocBiol 58:325-330. https://doi.org/10.1002/jlb.58.3.325

Ding S, Liang TJ (2020) Is SARS-CoV-2 also an enteric pathogen with potential fecal-oral transmission? A COVID-19 virological and clinical review. Gastroneterology 159:53-61. https://doi. org/10.1053/j.gastro.2020.04.052

Drucker DJ (2020a) Coronavirus infections and type 2 diabetes-shared pathways with therapeutic implications. Endocr Rev 41:bnaa011. https://doi.org/10.1210/endrev/bnaa011

Dubé MP, Chan ES, Lake JE et al (2019) A randomized, doubleblinded, placebo-controlled trial of sitagliptin for reducing inflammation and immune activation in treated and suppressed human immunodeficiency virus infection. Clin Infect Dis 69:1165-1172. https://doi.org/10.1093/cid/ciy1051

Fadini GP, Morieri ML, Longato E et al (2020) Exposure to DPP-4 inhibitors and COVID-19 among people with type 2 diabetes. DiabetesObesMetab, A case-control study. https://doi.org/10.1111/ dom.14097.10.1111/dom.14097

Gallwitz B (2019) Clinical Use of DPP-4 Inhibitors. Front Endocrinol 10:389. https://doi.org/10.3389/fendo.2019.00389

Gooßen K, Gräber S (2012) Longer term safety of dipeptidyl peptidase-4 inhibitors in patients with type 2 diabetes mellitus: systematic review and meta-analysis. Diabetes ObesMetab 14:10611072. https://doi.org/10.1111/j.1463-1326.2012.01610.x

Gorbalenya AE, Baker SC, Baric RS et al (2020) The species Severe Acute Respiratory Syndrome-related coronavirus: classifying 2019-nCoV and naming it SARS-CoV-2. Nat Microbiol 5:536544. https://doi.org/10.1038/s41564-020-0695-z

Hippisley-Cox J, Young D, Coupland C et al (2020) Risk of severe COVID-19 disease with ACE inhibitors and angiotensin receptor blockers: cohort study including 8.3 million people. Heart 106:1503-1511. https://doi.org/10.1136/heartjnl-2020-317393

Hoffman M, Kleine-Weber H, Schroeder S et al (2020) SARS-CoV-2 cell entry depends on ACE2 and TMPRSS2 and is blocked by a clinically proven protease inhibitor. Cell 181:271-280.e8. https:// doi.org/10.1016/j.cell.2020.02.052

Hussain A, Bhowmik B, do Vale Moreira NC (2020) COVID-19 and diabetes: knowledge in progress. Diabetes Res ClinPract 162:108142. https://doi.org/10.1016/j.diabres.2020.108142

Iacobellis G (2020) COVID-19 and diabetes: Can DPP4 inhibition play a role? Diabetes Res ClinPract 162:108125. https://doi. org/10.1016/j.diabres.2020.108125

Inn KS, Kim Y, Aigerim A et al (2018) Reduction of soluble dipeptidyl peptidase 4 levels in plasma of patients infected with middle east respiratory syndrome coronavirus. Virology 518:324-327. https ://doi.org/10.1016/j.virol.2018.03.015

Kaji K, Yoshiji H, Ikenaka Y et al (2014) Dipeptidyl peptidase-4 inhibitor attenuates hepatic fibrosis via suppression of activated hepatic stellate cell in rats. J Gastroenterol 49:481-491. https:// doi.org/10.1007/s00535-013-0783-4

Kim KM, Noh JH, Bodogai M et al (2017) Identification of senescent cell surface targetable protein DPP4. Genes Dev 31:1529-1534. https://doi.org/10.1101/gad.302570.117

Klemann C, Wagner L, Stephan M et al (2016) Cut to the chase: a review of CD26/dipeptidyl peptidase-4's (DPP4) entanglement in the immune system. ClinExpImmunol 185:1-21. https://doi. org/10.1111/cei.12781

Krejner-Bienias A, Grzela K, Grzela T (2020) do novel drugs for diabetes help in COVID-19? Another brick in the wall? J Diabetes 12:703-704. https://doi.org/10.1111/1753-0407.13050
Kulcsar KA, Coleman CM, Beck SE et al (2019) Comorbid diabetes results in immune dysregulation and enhanced disease severity following MERS-CoV infection. JCI Insight 4:e131774. https:// doi.org/10.1172/jci.insight.131774

Kupferschmidt K (2020) A cheap steroid is the first drug shown to reduce death in COVID-19 patients. Science. https://doi. org/10.1126/science.abd3683

Lambeir AM, Durinx C, Scharpe S et al (2003) Dipeptidyl-peptidase IV from bench to bedside: an update on structural properties, functions, and clinical aspects of the enzyme DPP IV. Crit Rev Clin Lab Sci 40:209-294. https://doi.org/10.1080/713609354

Lamers D, Famulla S, Wronkowitz N et al (2011) Dipeptidyl peptidase 4 is a novel adipokine potentially linking obesity to the metabolic syndrome. Diabetes 60:1917-1925. https://doi.org/10.2337/ db10-1707

Li W, Moore MJ, Vasilieva N et al (2003) Angiotensin-converting enzyme 2 is a functional receptor for the SARS coronavirus. Nature 426:450-454. https://doi.org/10.1038/nature02145

Li X, Geng M, Peng Y et al (2020) Molecular immune pathogenesis and diagnosis of COVID-19. J Pharm Anal 10:102-108. https:// doi.org/10.1016/j.jpha.2020.03.001

Li Y, Zhang Z, Yang L et al (2020b) The MERS-CoV receptor DPP4 as a candidate binding target of the SARS-CoV-2 Spike. iScience 23:101160. https://doi.org/10.1016/j.isci.2020.101160

Liu F, Li L, Xu M et al (2020) Prognostic value of interleukin-6, C-reactive protein, and procalcitonin in patients with COVID-19. J ClinVirol 127:104370. https://doi.org/10.1016/j.jcv.2020.104370

Lu R, Zhao X, Li J et al (2020) Genomic characterisation and epidemiology of 2019 novel coronavirus: implications for virus origins and receptor binding. Lancet 395:565-574. https://doi. org/10.1016/S0140-6736(20)30251-8

Makdissi A, Ghanim H, Vora M et al (2012) Sitagliptin exerts an antinflammatory action. J ClinEndocrinolMetab 97:3333-3341. https ://doi.org/10.1210/jc.2012-1544

Panchapakesan U, Pollock C (2015) The role of dipeptidyl peptidase-4 inhibitors in diabetic kidney disease. Front Immunol 6:443. https ://doi.org/10.3389/fimmu.2015.00443

Price JD, Linder G, Li WP et al (2013) Effects of short-term sitagliptin treatment on immune parameters in healthy individuals, a randomized placebo-controlled study. ClinExpImmunol 174:120-128. https://doi.org/10.1111/cei.12144

Raj VS, Mou H, Smits SL et al (2013) Dipeptidyl peptidase 4 is a functional receptor for the emerging human coronavirus-EMC. Nature 495:251-254. https://doi.org/10.1038/nature12005

Raj VS, Smits SL, Provacia LB et al (2014) Adenosine deaminase acts as a natural antagonist for dipeptidyl peptidase 4-mediated entry of the Middle East Respiratory Syndrome coronavirus. J Virol 88:1834-1838. https://doi.org/10.1128/JVI.02935-13

Röhrborn D, Wronkowitz N, Eckel J (2015) DPP4 in diabetes. Front Immunol 6:386. https://doi.org/10.3389/fimmu.2015.00386

Roncon L, Zuin M, Rigatelli G et al (2020) Diabetic patients with COVID-19 infection are at higher risk of ICU admission and poor short-term outcome. J ClinVirol 127:104354. https://doi. org/10.1016/j.jcv.2020.104354

Scheen AJ, Marre M, Thivolet C (2020) Prognostic factors in patients with diabetes hospitalized for COVID-19: Findings from the CORONADO study and other recent reports. Diabetes Metab 46:265-271. https://doi.org/10.1016/j.diabet.2020.05.008

Schlicht K, Rohmann N, Geisler C et al (2020) Circulating levels of soluble dipeptidylpeptidase- 4 are reduced in human subjects hospitalized for severe COVID-19 infections. Int J Obes 44:23352338. https://doi.org/10.1038/s41366-020-00689-y

Sesti G, Avogaro A, Belcastro S et al (2019) Ten years of experience with DPP-4 inhibitors for the treatment of type 2 diabetes mellitus. ActaDiabetol 56:605-617. https://doi.org/10.1007/s0059 2-018-1271-3 
Seys LJM, Widagdo W, Verhamme FM et al (2018) DPP4, the Middle East Respiratory Syndrome Coronavirus receptor, is upregulated in lungs of smokers and chronic obstructive pulmonary disease patients. Clin Infect Dis 66:45-53. https://doi.org/10.1093/cid/ cix741

Smith RE, Talhouk JW, Brown EE et al (1998) The significance of hypersialylation of dipeptidyl peptidase IV (CD26) in the inhibition of its activity by tat and other cationic peptides. CD26: a subverted adhesion molecule for HIV peptide binding. AIDS Res Hum Retroviruses 14:851-868. https://doi.org/10.1089/ aid.1998.14.851

Solerte SB, Di Sabatino A, Galli M et al (2020a) Dipeptidyl peptidase-4 (DPP4) inhibition in COVID-19. ActaDiabetol 57:779_ 783. https://doi.org/10.1007/s00592-020-01539-z

Solerte SB, D'Addio F, Trevisan R et al (2020b) Sitagliptin treatment at the time of hospitalization was associated with reduced mortality in patients with type 2 diabetes and COVID-19: A multicenter, case-control, retrospective, observational study. Diabetes Care 43:2999-3006. https://doi.org/10.2337/dc20-1521

Sromova L, Busek P, Posova H et al (2016) The effect of dipeptidyl peptidase-IV inhibition on circulating T cell subpopulations in patients with type 2 diabetes mellitus. Diabetes Res ClinPract 118:183-192. https://doi.org/10.1016/j.diabres.2016.06.020

Takagaki Y, Shi S, Katoh M et al (2019) Dipeptidyl peptidase-4 plays a pathogenic role in BSA-induced kidney injury in diabetic mice. Sci Rep 9:7519. https://doi.org/10.1038/s41598-019-43730-5

Vankadari N, Wilce JA (2020) Emerging WuHan (COVID-19) coronavirus: glycan shield and structure prediction of spike glycoprotein and its interaction with human CD26. Emerg Microbes Infect 9:601-604. https://doi.org/10.1080/22221751.2020.1739565

Vora KA, Porter G, Peng R et al (2009) Genetic ablation or pharmacological blockade of dipeptidyl peptidase IV does not impact $\mathrm{T}$ cell-dependent immune responses. BMC Immunol 10:19. https:// doi.org/10.1186/1471-2172-10-19
World Health Organization (2020) Coronavirus disease 2019 (COVID19): situation report; https://www.worldometers.info/coronavirus. Accessed Nov 24th, 2020

Wvan der Zanden R, de Vries F, Lalmohamed A et al (2015) Use of dipeptidyl-peptidase-4 inhibitors and the risk of pneumonia: a population-based cohort study. PLoS ONE 10:e0139367. https:// doi.org/10.1371/journal.pone.0139367

Xi CR, Di Fazio A, Nadvi NA et al (2020) A novel purification procedure for active recombinant human DPP4 and the inability of DPP4 to bind SARS-CoV-2. Molecules 25:5392. https://doi. org/10.3390/molecules25225392

Yang J, Zheng Y, Gou X et al (2020) Prevalence of comorbidities and its effects in patients infected with SARS-CoV-2: A systematic review and meta-analysis. Int J Infect Dis 94:91-95. https://doi. org/10.1016/j.ijid.2020.03.017

Yang W, Cai X, Han X et al (2016) DPP-4 inhibitors and risk of infections: a meta-analysis of randomized controlled trials. Diabetes Metab Res Rev 32:391-404. https://doi.org/10.1002/dmrr.2723

Yu DMT, Slaitini L, Gysbers V et al (2011) Soluble CD26 / dipeptidyl peptidase IV enhances human lymphocyte proliferation in vitro independent of dipeptidyl peptidase enzyme activity and adenosine deaminase binding. Scand J Immunol 73:102-111. https:// doi.org/10.1111/j.1365-3083.2010.02488.x

Zhao Q, Meng M, Kumar R, Wu Y et al (2020) Lymphopenia is associated with severe coronavirus disease 2019 (COVID-19) infections: A systemic review and meta-analysis. Int J Infect Dis 96:131-135. https://doi.org/10.1016/j.ijid.2020.04.086

Publisher's Note Springer Nature remains neutral with regard to jurisdictional claims in published maps and institutional affiliations. 